\title{
Every Graph Admits an Unambiguous Bold Drawing
}

\author{
János Pach* \\ EPFL, Lausanne and Rényi Institute, Budapest \\ pach@cims.nyu.edu
}

\begin{abstract}
Let $r$ and $w$ be a fixed positive numbers, $w<r$. In a bold drawing of a graph, every vertex is represented by a disk of radius $r$, and every edge by a narrow rectangle of width $w$. We solve a problem of van Kreveld K09] by showing that every graph admits a bold drawing in which the region occupied by the union of the disks and rectangles representing the vertices and edges does not contain any disk of radius $r$ other than the ones representing the vertices.
\end{abstract}

\section{Introduction}

In this note, we adopt a "realistic" view of graph drawing, proposed by Marc van Kreveld [K09]. Let $G$ be a graph with vertices $v_{1}, \ldots, v_{n}$, represented by points in the plane, and let the edges be drawn as possibly crossing straightline segments. Now fix two positive numbers $r$ and $w, w<2 r$, and replace each vertex by a disk of radius $r$ centered at $v_{i}$, and each edge $v_{i} v_{j}$ by a rectangle such that its midsegment is $v_{i} v_{j}$ and its width, the length of its side perpendicular to $v_{i} v_{j}$, is $w$. We call the union $D$ of these disks and rectangles a bold drawing of $G$. A bold drawing is said to be unambiguous if it satisfies the following two conditions.

1. No two disks representing vertices of $G$ intersect.

2. The set $D$ contains no disk of radius $r$ other than the disks representing its vertices.

The first condition is equivalent to saying that $2 r$ is smaller than the minimum distance between two points $v_{i}$ and $v_{j}$. It follows from the second condition that a bold drawing of a graph which has at least one edge can be unambiguous only if $w<2 r$. It was shown in $\mathrm{K} 09$ that if $w>r$, then the maximum degree of the vertices of all graphs that admit an unambiguous bold drawing is bounded from above by a constant depending only on $w$ and $r$. On the other hand, van Kreveld proved that for $w<r$, any star consisting of a central vertex connected to an arbitrary number of other vertices admits an unambiguous bold drawing.

\footnotetext{
* Supported by NSF Grant CCF-08-30272, by NSA, by OTKA under EUROGIGA project GraDR 10-EuroGIGA-OP-003, and by Swiss National Science Foundation Grant 200021-125287/1.
} 
He also raised the question whether there exists a fixed pair of values $w, r$ with $w<r$ such that with these parameters every finite graph admits an unambiguous bold drawing. The aim of this note is to answer this question in the affirmative in the following strong sense.

Theorem 1. Let $w$ and $r$ be any positive constants with $w<r$. Then, for every positive integer $n$, the complete graph $K_{n}$ admits an unambiguous bold drawing, in which the vertices are represented by disks of radius $r$ and the edges by rectangles of width $w$.

In the next statement, we describe our construction in full detail.

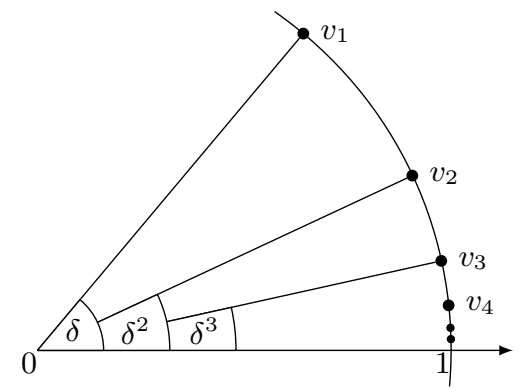

Fig. 1. Construction for Theorem 2

Theorem 2. Let $w$ and $r$ be any positive constants with $w<r$. Let $C$ be a circle of radius 1 around the origin, and let $v_{i}(1 \leq i \leq n)$ denote the intersection point of $C$ and the ray obtained from the positive $x$-axis by a counterclockwise rotation through angle $\delta^{i}$, where $\delta=\min \left(\frac{1}{2}, 1-\frac{w}{r}\right)$.

For every $n$, there exists a sufficiently small $\varepsilon=\varepsilon(n)>0$ such that replacing each $v_{i}$ by a disk of radius $\varepsilon r$ centered at $v_{i}$ and each edge $v_{i} v_{j}$ by a rectangle of width $\varepsilon w$ with midsegment $v_{i} v_{j}$, the union of these disks and rectangles contains no disk of radius $\varepsilon r$ other than the ones representing the vertices.

Theorem 2 immediately implies Theorem 1. Indeed, if we choose $\varepsilon(n)>0$ so small that in addition to the property in Theorem 2, it satisfies the inequality $2 \varepsilon r<\min _{1 \leq i<j \leq n}\left|v_{i} v_{j}\right|=\left|v_{n-1} v_{n}\right|$, and we blow up the drawing described in Theorem 2 by a factor of $1 / \varepsilon$, then we obtain a bold drawing of $K_{n}$ that meets both requirements for unambiguity stated above.

In K09, van Kreveld listed seven properties that a "good" bold drawing of a graph $G$ must satisfy. These include the two conditions for unambiguous drawings stated above, so that every good bold drawing of $G$ is also unambiguous. It is easy to see that if we choose the constant $\varepsilon(n)$ small enough, then our drawing of $K_{n}$ will also meet the five additional properties formulated in [K09].

Before turning to the proof, we would like to argue that in some sense we are "forced" to consider constructions of the type described in Theorem 2 We say 
that a set of points in the plane is in general position if no three of them are collinear. According to the Erdös-Szekeres theorem [ES35], for any integer $K$, every sufficiently large set of points in general position in the plane contains $K$ elements that form the vertex set of a convex $K$-gon. This readily implies, that for any $K$ there exists $N(K)$ such that any set of $N(K)$ points in general position has $K$ elements that lie on a convex curve whose total turning angle is small. By rotating the coordinate axes if necessary, the coordinates of these points can be written as $\left(x_{i}, f\left(x_{i}\right)\right)$, where $x_{1}<x_{2}<\ldots<x_{K}$ and $f(x)$ is a smooth convex function whose derivative is bounded by a small constant. Let $\gamma=\frac{\sqrt{5}+1}{2} \approx 1.618$, the golden ratio. Color the triples $(i, j, k), 1 \leq i<j<k \leq K$, with red, blue, or green, according to whether $\frac{x_{k}-x_{j}}{x_{j}-x_{i}}$ is at most $\gamma^{-1}$, belongs to the interval $\left(\gamma^{-1}, \gamma\right)$, or is at least $\gamma$, respectively. According to Ramsey's theorem [R30, GRS90, for every $n \geq 4$ we can choose $K=K(n)$ so large that there is a sequence $1 \leq i_{1}<i_{2}<\ldots<i_{n} \leq K$ with the property that all triples determined by its members are of the same color. It is easy to check that there exists no sequence of length 4 such that all of its triples are blue. Therefore, we can assume that all triples determined by the sequence $1 \leq i_{1}<i_{2}<\ldots<i_{n} \leq K$ are red or all of them are green. In the first case the distances $x_{i+1}-x_{i}$ decrease, in the second one increase at least exponentially fast, as $i$ grows $(1 \leq i \leq n)$. Summarizing: for every $n \geq 4$, there is an integer $N$ with the property that from any set of $N$ points in general position in the plane we can select a sequence of length $n$ which lies on an arc of a convex curve with small total turning angle and the distances between its consecutive elements decrease at least exponentially. (We can reverse the numbering of the elements, if necessary.) Suppose now that $K_{N}$ admits an unambiguous bold drawing. Applying the last statement to the centers of the disks representing the vertices, we obtain an unambiguous bold subdrawing of a complete graph $K_{n}$ such that the centers of the disks representing its vertices lie on a convex curve and the distances between them are fast decreasing. Our construction in Theorem 2 is motivated by this observation.

The proof of Theorem 2 is somewhat subtle. In Sect. 2, we introduce some definitions that simplify the presentation and we state two easy but useful lemmas that can be proved by direct computation. The heart of the proof lies in Lemma 5, stated and established in Sect. 3. After this preparation, the proof of Theorem 2 presented in Sect. 4 is rather straightforward.

Several graph drawing programs for straight-line drawing offer the option to draw the vertices and the edges bold (see, for example, NEATO N04]). Some algorithmic aspects of bold drawing were addressed in [K09]. In particular, given a drawing of a graph $G$ with possibly crossing straight-line edges, van Kreveld applied a line segment intersection algorithm [CE92, CS89], [M88, to find the smallest $w$ for which, if we draw the edges as closed rectangles of width $w$, we find three edges, not all incident to the same vertex, such that the corresponding rectangles have a point in common. Duncan, Efrat, Kobourov, and Wenk DEKW06] presented an efficient algorithm to determine the largest $w$, 
for a given planar embedding of a graph $G$, such that $G$ admits an equivalent drawing in which the edges are represented by nonoverlapping, not necessarily straight bold curves of width $w$.

\section{Terminology and Two Preliminary Lemmas}

In the rest of this note, $w$ and $r$ are fixed positive numbers with $w<r$. Throughout the next two sections, we also fix the parameter $\varepsilon>0$, which will be varied only in Sect. 4, in the proof of Theorem 2 .

First, we introduce some notation and terminology. Let $v$ be a point of the plane, and let $R_{1}, \ldots, R_{s}$ be a set of infinite rays (half-lines) emanating from $v$, listed in clockwise order. Assume that all rays $R_{i}$ point into the same half-plane bounded by a line passing through $v$. Replace $v$ by a closed disk of radius $\varepsilon r$ centered at $v$, and replace each $R_{i}$ by a closed one-way infinite half-strip of width $\varepsilon w$ with $R_{i}$ as its mid-ray. The union of the disk and these half-strips is called a palm and is denoted by $P=P\left(v, R_{1}, \ldots, R_{s}\right)$. The point $v$ is said to be the apex of the palm, the half-strips are said to be its fingers, and the largest angle between the rays defining two (not necessarily consecutive) fingers is the angle of the palm.

If we go far enough from $v$, the fingers start to bifurcate. For any two consecutive fingers corresponding to the rays $R_{i}$ and $R_{i+1}$, we define the distance from $v$ at which they bifurcate, as the maximum radius of a disk centered at $v$ with the property that its intersection with the complement of the union of the fingers (half-strips) is connected. Analogously, for any two (two-way infinite) strips $S$ and $S^{\prime}$ such that their midlines cross at a point $v$, we define the distance from $v$ at which they bifurcate as the maximum radius of a disk centered at $v$ with the property that its intersection with the complement of $S \cup S^{\prime}$ has at most two connected components.

The following two simple statements can be established by straightforward trigonometric calculations.

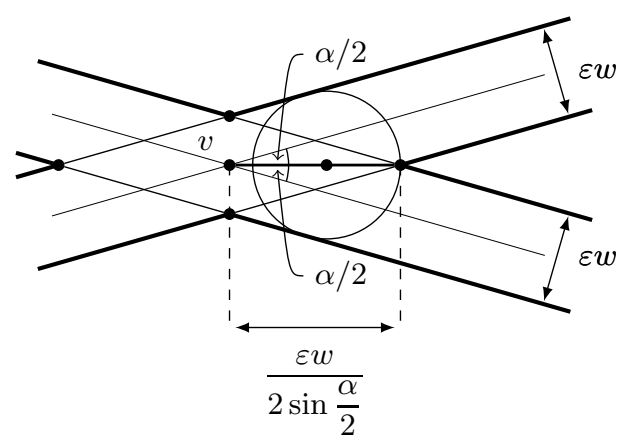

Fig. 2. For Lemma 3 
Lemma 3. Let $S$ and $S^{\prime}$ be two strips of width $\varepsilon w$ such that their midlines cross at a point $v$ and the angle between them is $\alpha \leq \frac{\pi}{2}$. Then

1. $S \cup S^{\prime}$ contains no disk of radius $\varepsilon w$;

2. $S$ and $S^{\prime}$ bifurcate at distance $\frac{\varepsilon w}{2 \sin \frac{\alpha}{2}}$ from $v$;

3. any two consecutive fingers of a palm such that the angle between the rays defining them is $\alpha \leq \pi / 2$ bifurcate at distance $\frac{\varepsilon w}{2 \sin \frac{\alpha}{2}}$ from the apex.

Lemma 4. Let $P=P\left(v, R_{1}, \ldots, R_{s}\right)$ be a palm as above, and assume that its angle is smaller than $2 \arcsin \frac{1}{4}<\frac{\pi}{6}$. Let $\bar{P} \supset P$ denote the union of the disk of radius $\varepsilon r$ centered at $v$ and the convex hull of the union of the first and last fingers, corresponding to $R_{1}$ and $R_{s}$.

Then $\bar{P}$ contains no disk of radius $\varepsilon r$ that intersects the disk of radius $\varepsilon r$ centered at its apex $v$. Hence, the same is true for $P$.

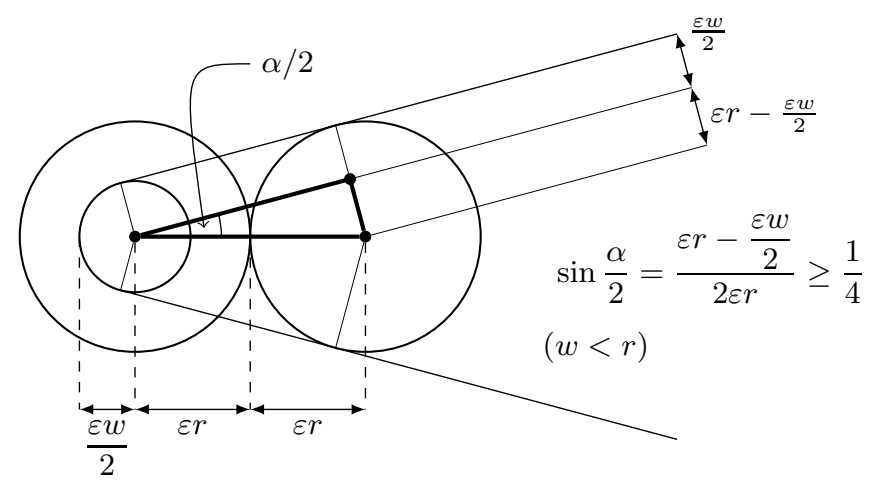

Fig. 3. For Lemma 4

\section{The Main Lemma}

As in the previous section, $w, r$, and $\varepsilon$ are fixed positive constants, $w<r$. The main component of the proof of Theorem 2 is the following lemma, which guarantees that if the angles between the consecutive fingers of a palm $P$ decrease sufficiently fast, then $P$ cannot contain a disk of radius $\varepsilon r$. The proof of this fact requires some detailed calculations, but heuristically it is clear that in this case only the first two fingers play an important role, and the situation is similar to the setting of Lemma 3 , part 1.

Lemma 5. Let $\delta=\min \left(\frac{1}{2}, 1-\frac{w}{r}\right)$, and let $P=P\left(v, R_{1}, \ldots, R_{s}\right)$ be a palm of angle $\alpha<\delta^{1 / 2}$. Let $\alpha_{i}$ denote the angle between $R_{i}$ and $R_{i+1}$, and assume that for every $i(1 \leq i<s)$ we have $\frac{\alpha_{i+1}}{\alpha_{i}} \leq \delta$.

Then $P$ contains no disk of radius $\varepsilon r$. 


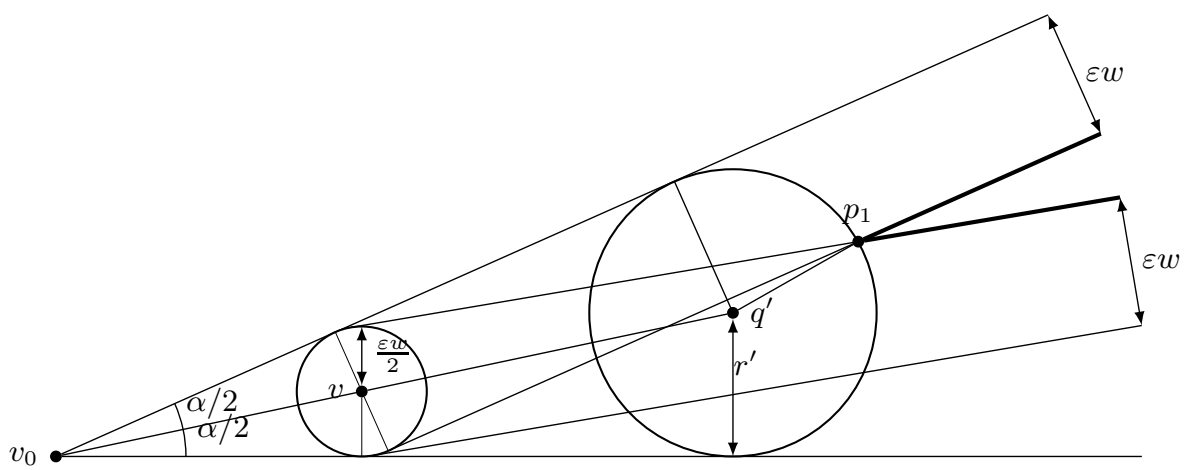

(a)

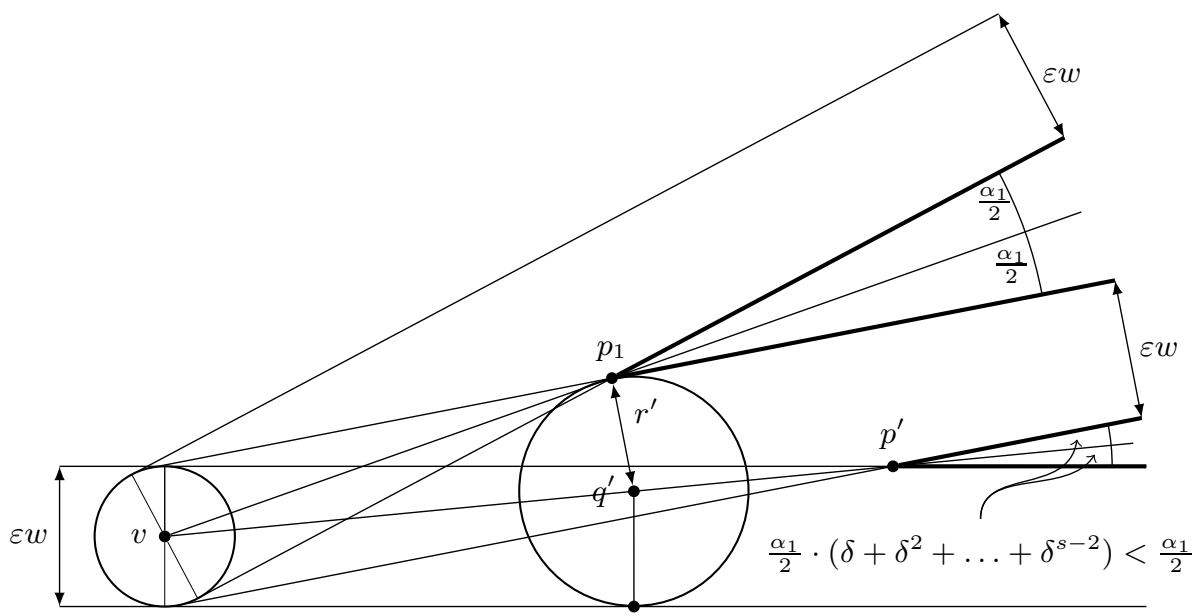

(b)

Fig. 4. For Lemma 5

Proof. If the fingers corresponding to $R_{i}$ and $R_{i+1}$ bifurcate at distance $d_{i}$ from $v$, then they share a boundary point $p_{i}$ with $\left|v p_{i}\right|=d_{i}(1 \leq i<s)$. These points are called points of bifurcation. It follows from the condition in Lemma 5 about the ratios $\alpha_{i+1} / \alpha_{i}$ that $d_{1}<d_{2}<d_{3}<\ldots$ is a fast increasing sequence. If $P$ has at most 2 fingers, then Lemma 5 is true by Lemma 3 , part 1 . Therefore, we can assume that $s$, the number of fingers, is at least 3 and that we have already proved the lemma for all palms with fewer than $s$ fingers.

Suppose that $\left|v p_{1}\right|=d_{1}=\min _{1 \leq i<s} d_{i} \leq \varepsilon r$. Then $P$ is the union of two palms $P\left(v, R_{1}\right)$ and $P\left(v, R_{2}, \ldots, R_{s}\right)$, each having fewer than $s$ fingers, so that any disk of radius $\varepsilon r$ other than the one centered at $v$ must belong to one of them. Thus, in this case we are done, by induction. From now on assume that $p_{1}$ and hence all other points $p_{i}$ lie outside of the disk of radius $\varepsilon r$ centered at $v$. Note that the part of the ray $v p_{i}$ beyond the point $p_{i}$ does not belong to $P$. 
In fact, it lies in an infinite open cone $C_{i}$, symmetric about $v p_{i}$, which belongs to the complement of $P$. By rotating the coordinate system if necessary, we can assume without loss of generality that $R_{s}$ is parallel to the positive $x$-axis, so that all other rays $R_{1}, \ldots, R_{s-1}$ point into the positive quadrant $x, y \geq 0$. Then it makes sense to talk about the lower and the upper boundary of a finger. The cone $C_{i}$ is bounded by two half-lines: one belongs to the lower boundary of the finger corresponding to $R_{i}$ and the other to the upper boundary of the finger corresponding to $R_{i+1}$.

Suppose for contradiction that $P$ contains a disk $D$ of radius at least $\varepsilon r$, other than the disk of radius $\varepsilon r$ centered at $v$. It follows from Lemma 4 that $D$ cannot intersect the disk of radius $\varepsilon r$ centered at $v$. We also know that $D$ must have a point that belongs only to the first finger, but not to the second one, otherwise we can remove the first finger and obtain a contradiction using the induction hypothesis.

Let $\bar{P}$ be the same as in Lemma 4 , and let $P^{\prime} \supset P$ denote the region obtained from $\bar{P}$ by deleting all points that belong to the infinite cone $C_{1}$ with apex $p_{1}$. Let $D^{\prime}$ be a disk of maximum radius in $P^{\prime}$ with the property that it has a point that belongs to the first finger of $P$, but not to the interior of the second one. Let $q^{\prime}$ and $r^{\prime}$ denote the center and the radius of $D^{\prime}$. By our assumption, we have that $r^{\prime} \geq \varepsilon r$, and it follows from Lemma 4 that $D^{\prime}$ does not intersect the disk of radius $\varepsilon r$ centered at $v$.

It is easy to verify that

1. $p_{1}$ lies on the boundary of $D^{\prime}$;

2. $D^{\prime}$ is tangent to the lower (horizontal) boundary half-line of $\bar{P}$;

3. $D^{\prime}$ is tangent either to the upper boundary half-line of the second finger or to the upper boundary half-line of $\bar{P}$.

Indeed, it follows from the maximality of $D^{\prime}$ that $D^{\prime}$ is "fixed" by the boundary of $P^{\prime}$. One point cannot fix a disk. The same is true for two points, one lying on the lower, one on the upper boundary half-line of $\bar{P}$. In other words, if $D^{\prime}$ is tangent to the lower and to the upper boundary half-lines of $\bar{P}$, by maximality, it must also touch the boundary of the cone $C_{i}$. Suppose first that $D^{\prime}$ is tangent to the upper boundary half-line of $\bar{P}$ and to the upper boundary half-line of $C_{1}$. If condition 1 is not satisfied, that is, $D^{\prime}$ touches a point of the upper boundary half-line of $C_{1}$ other than $p_{1}$, then $D^{\prime}$ must lie entirely in the first finger, and its radius cannot exceed $\varepsilon w / 2<\varepsilon r$, which is impossible. Therefore, condition 1 is satisfied and, unless $D^{\prime}$ also satisfies condition $2, D^{\prime}$ can be enlarged without violating the requirements.

Suppose next that $D^{\prime}$ is not tangent to the upper boundary half-line of $\bar{P}$. Then $D^{\prime}$ must be tangent to the lower boundary half-line of $\bar{P}$ and to the lower boundary half-line of $C_{1}$. Moreover, the point at which $D^{\prime}$ touches the lower boundary half-line of $C_{1}$ must be $p_{1}$, otherwise $D^{\prime}$ cannot have a point that belongs to the first finger of $P$, but not to the interior of the second one. If $D^{\prime}$ has such a point strictly above the upper boundary of the second finger then it could be slightly enlarged without violating the conditions. Indeed, $q^{\prime}$ belongs to the locus of all points equidistant from $p_{1}$ and the (horizontal) supporting line 
of the lower boundary half-line of $\bar{P}$, which is a parabola $\Pi$ with a vertical axis of symmetry. If $q^{\prime}$ is on the left side of this parabola, then we can enlarge the radius of $D^{\prime}$ by moving $q^{\prime}$ along $\Pi$ slightly to the left, if it is on the right side of $\Pi$, then by moving it slightly to the right. Therefore, we can conclude that $D^{\prime}$ must be tangent to the upper boundary of the second finger at point $p_{1}$, and condition 3 holds.

Now we can easily complete the proof of Lemma 5 .

If conditions 1,2 , and the first option in condition 3 hold, then consider the triangle $v p_{1} q^{\prime}$. Using that $\delta \leq 1 / 2$, we obtain

$$
\begin{aligned}
\angle v p_{1} q^{\prime} & =\frac{\pi}{2}-\frac{\alpha_{1}}{2} \leq \frac{\pi}{2}-\frac{\delta \alpha_{1}}{2(1-\delta)} \\
& <\frac{\pi}{2}-\frac{\alpha_{1}}{2}\left(\delta+\delta^{2}+\ldots+\delta^{s-2}\right) \\
& \leq \frac{\pi}{2}-\frac{\alpha_{2}+\alpha_{3}+\ldots+\alpha_{s-1}}{2}=\angle v q^{\prime} p_{1}
\end{aligned}
$$

This yields that $\left|v q^{\prime}\right|<\left|v p_{1}\right|$. As was used above, the angle $\alpha_{1}$ between $R_{1}$ and $R_{2}$ is larger than $\alpha_{2}+\ldots+\alpha_{s-1}$, the angle between $R_{2}$ and $R_{s}$. Therefore, the fingers corresponding to $R_{2}$ and $R_{s}$ bifurcate at a point $p^{\prime}$ which is farther away from $v$ than $p_{1}$ is. This implies that $\left|v q^{\prime}\right|<\left|v p_{1}\right|<\left|v p^{\prime}\right|$. The points $v, q^{\prime}$, and $p^{\prime}$ are collinear, so that it follows from the last inequality that $q^{\prime}$ lies in the interior of the second finger. Since $r^{\prime}=\left|q^{\prime} p_{1}\right|$ is equal to the distance of $q^{\prime}$ from the upper boundary half-line of the second finger, we obtain that $r^{\prime}<\varepsilon w<\varepsilon r$, which is a contradiction.

In the other case, when conditions 1,2 , and the second option in condition 3 hold, just like in the first case, we have $\left|v q^{\prime}\right|<\left|v p_{1}\right|$. (In fact, it is easy to argue that the part of the parabola $\Pi$ which lies below the line $v p_{1}$ and to the left of the line through $p_{1}$ perpendicular to $R_{s}$ is entirely contained in the interior of the circle through $p_{1}$ centered at $v$. The point $q^{\prime}$ belongs to this arc.)

Let $v_{0}$ denote the intersection point of the supporting lines of the upper boundary ray of the first finger (that corresponds to $R_{1}$ ) and the lower boundary ray of the last finger (that corresponds to $R_{s}$ ). The points $v_{0}, v$, and $q^{\prime}$ are collinear. Using the notation $\alpha=\alpha_{1}+\ldots+\alpha_{s-1}$, we have

$$
\begin{aligned}
r^{\prime} & =\left|v_{0} q^{\prime}\right| \sin \frac{\alpha}{2}=\left(\left|v_{0} v\right|+\left|v q^{\prime}\right|\right) \sin \frac{\alpha}{2}<\left(\left|v_{0} v\right|+\left|v p_{1}\right|\right) \sin \frac{\alpha}{2} \\
& \leq\left(\frac{\varepsilon w}{2 \sin \frac{\alpha}{2}}+\frac{\varepsilon w}{2 \sin \frac{\alpha_{1}}{2}}\right) \sin \frac{\alpha}{2}=\frac{\varepsilon w}{2}\left(1+\frac{\sin \frac{\alpha}{2}}{\sin \frac{\alpha_{1}}{2}}\right) .
\end{aligned}
$$

Here we used Lemma 3, part 2 to estimate $\left|v p_{1}\right|$.

In view of the assumption on the angles between consecutive fingers, we have that

$$
\alpha=\alpha_{1}+\alpha_{2}+\ldots+\alpha_{s-1}=\alpha_{1}\left(1+\delta+\ldots+\delta^{s-2}\right)<\frac{\alpha_{1}}{1-\delta} .
$$


Hence, the above upper bound on $r^{\prime}$ can be rewritten as

$$
r^{\prime}<\frac{\varepsilon w}{2}\left(1+\frac{\sin \frac{\alpha_{1}}{2(1-\delta)}}{\sin \frac{\alpha_{1}}{2}}\right)<\frac{\varepsilon w}{2}\left(1+\frac{\frac{\alpha_{1}}{2(1-\delta)}}{\sin \frac{\alpha_{1}}{2}}\right)
$$

Using the Taylor series of the $\sin x$ function, it is easy to verify that, given any $\delta, 0<\delta<1$, the inequality $\sin \frac{\alpha_{1}}{2}>\frac{\alpha_{1}}{2(1+\delta)}$ holds for all $\alpha_{1} \leq \delta^{1 / 2}$. By the assumptions in the lemma, this condition is satisfied, so that we have

$$
r^{\prime}<\frac{\varepsilon w}{2}\left(1+\frac{1+\delta}{1-\delta}\right)=\frac{\varepsilon w}{1-\delta} .
$$

By our choice of $\delta$, we have $\delta \leq 1-\frac{w}{r}$. That is,

$$
r^{\prime}<\frac{\varepsilon w}{1-\delta} \leq \varepsilon r
$$

the desired contradiction.

\section{The Proof of Theorem 2}

In the previous two sections, apart from $n, w$, and $r$, we also fixed the constant $\varepsilon>0$. In the proof of Theorem 2 presented in this section, we keep $n, w$, and $r$ fixed, but we will vary $\varepsilon$.

Let $S(\varepsilon)$ denote the union of the disks of radius $\varepsilon r$ representing the vertices $v_{i}(1 \leq i \leq n)$ and the rectangles of width $\varepsilon w$ representing the edges $v_{i} v_{j}(1 \leq$ $i<j \leq n)$.

For a given $v_{i}$, consider the rectangles representing the edges incident to $v_{i}$ and extend them to one-way infinite half-strips pointing away from $v_{i}$. More precisely, for any $j \neq i$, let $R_{i, j}$ denote the ray $\overrightarrow{v_{i} v_{j}}$ emanating from $v_{i}$ and pointing to the direction of $v_{j}$. Let $F_{i, j}(\varepsilon)$ be the half-strip of width $\varepsilon w$, the mid-ray of which is $R_{i, j}$. The union of the disk of radius $\varepsilon r$ centered at $v_{i}$ and the sets $F_{i, j}(\varepsilon)$ for all $j \neq i$ is denoted by $P_{i}(\varepsilon)$. Any two distinct half-strips $F_{i, j}(\varepsilon)$ and $F_{i, j^{\prime}}(\varepsilon)$ bifurcate at a certain distance from $v_{i}$. Let $\varrho_{i}(\varepsilon)$ denote the maximum of these $\left(\begin{array}{c}n-1 \\ 2\end{array}\right)$ distances plus $\varepsilon r$.

Let us fix a small $\varepsilon>0$ such that the following three conditions are satisfied.

1. No three rectangles representing distinct edges, not all of which are incident to the same vertex, have a point in common.

2. Any rectangle representing an edge $v_{j} v_{k}$ is disjoint from any disk of radius $\varrho_{i}(\varepsilon)$ centered at $v_{i}$, for all $i \neq j, k$.

3. For every pair $i \neq j$, the disk of radius $\varrho_{i}(\varepsilon)$ centered at $v_{i}$ is disjoint from the disk of radius $\varrho_{j}(\varepsilon)$ centered at $v_{j}$.

It follows from the second condition that no rectangle representing an edge $v_{j} v_{k}$ can intersect any disk representing a vertex $v_{i}$ with $i \neq j, k$. The last condition implies that the disk of radius $\varrho_{i}(\varepsilon)$ centered at $v_{i}$ cannot contain any disk of 


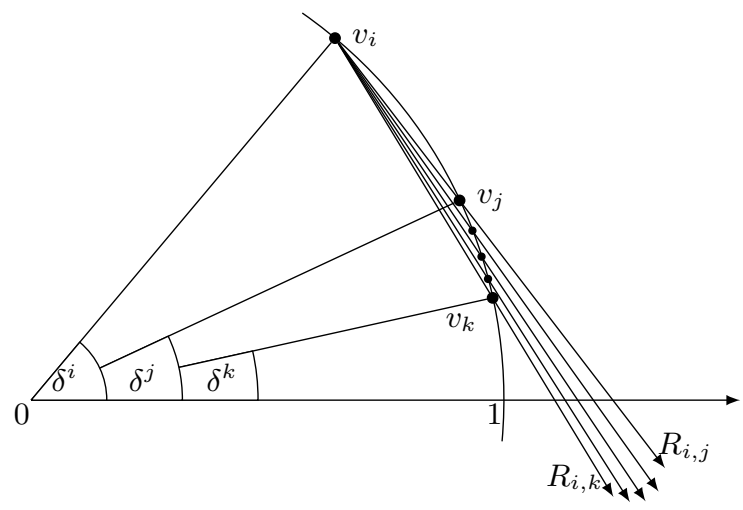

Fig. 5. For Proof of Theorem 2

radius $\varepsilon r$ representing a vertex $v_{j}$ with $j \neq i$. If three edges share an interior point, then the first condition cannot be satisfied. However, it is easy to argue that in our case this cannot occur.

From now on $\varepsilon$ will be fixed, so that in notation we can drop the parameter $\varepsilon$. In particular, instead of $S(\varepsilon), \varrho_{i}(\varepsilon)$, and $P_{i}(\varepsilon)$, we will write $S, \varrho_{i}$, and $P_{i}$.

Suppose for contradiction that the set $S$ contains a disk $D$ of radius $\varepsilon r$ which is not one of the disks representing the vertices. Where can such a disk $D$ lie? The only possibility is that for some $i(1 \leq i \leq n)$, it lies in the part of $S$ contained in the disk of radius $\varrho_{i}$ centered at $v_{i}$. Otherwise, by the conditions listed above, $D$ would be contained in the union of two strips of width $w$, contradicting part 1 of Lemma 3. Observe that the part of $S$ contained in the disk of radius $\varrho_{i}$ centered at $v_{i}$ is exactly the same as the part of $P_{i}$ contained in the disk of radius $\varrho_{i}$ centered at $v_{i}$. Therefore, to finish the proof of Theorem 2, it is sufficient to show that no set $P_{i}$ contains a disk of radius $\varepsilon r(1 \leq i \leq n)$.

To see this, notice that for every $i 1 \leq i \leq n$, the set $P_{i}$ can be written as the union of at most two palms of angle smaller than $\delta$ (see the beginning of Sect. 2). We have $P_{1}=P\left(v_{1}, R_{1,2}, R_{1,3}, \ldots, R_{1, n}\right), P_{n}=P\left(v_{n}, R_{n, 1}, R_{n, 2}, \ldots, R_{n, n-1}\right)$, and

$$
P_{i}=P\left(v_{i}, R_{i, 1}, R_{i, 2}, \ldots, R_{i, i-1}\right) \cup P\left(v_{i}, R_{i, i+1}, R_{i, i+2}, \ldots, R_{i, n}\right),
$$

for every $i, 1<i<j$. If $i \neq 1, n$, then the smallest angle between a finger of $P\left(v_{i}, R_{i, 1}, R_{i, 2}, \ldots, R_{i, i-1}\right)$ and a finger of $P\left(v_{i}, R_{i, i+1}, R_{i, i+2}, \ldots, R_{i, n}\right)$ is the angle between $R_{i, 1}$ and $R_{i, n}$, which is equal to $\pi-\frac{\delta-\delta^{n}}{2}>\pi-\frac{\delta}{2}$. It follows from here that the fingers corresponding to $R_{i, 1}$ and $R_{i, n}$ bifurcate within the disk of radius $\varepsilon r$ centered at $v_{i}$. This, in turn, implies that any disk $D$ of radius $\varepsilon r$ which lies in $P_{i}$ and is different from the disk representing $v_{i}$ is entirely contained in one of the two palms comprising $P_{i}$. Applying Lemma 5 to this palm, we obtain the desired contradiction. The only thing that remains to be checked is that the conditions of the lemma about the angles $\alpha$ and $\alpha_{i}$ are satisfied. 
The maximum angle of the palms of the form $P\left(v_{i}, R_{i, i+1}, R_{i, i+2}, \ldots, R_{i, n}\right)$ and $P\left(v_{i}, R_{i, 1}, R_{i, 2}, \ldots, R_{i, i-1}\right)$, for $1 \leq i \leq n$, is the angle of $P\left(v_{n}, R_{n, 1}, R_{n, 2}, \ldots, R_{n, n-1}\right)$, which is equal to

$$
\angle v_{1} v_{n} v_{n-1}=\frac{\angle v_{1} 0 v_{n-1}}{2}=\frac{\delta-\delta^{n-1}}{2}<\frac{\delta}{2},
$$

so that the condition on the angle of the palm is satisfied. (Here 0 denotes the origin, the center of the circle containing all points $v_{i}$.) As for the condition on the angles $\alpha_{i}$, we have that the angle between two consecutive rays $R_{i, t}$ and $R_{i, t+1}$ is equal to

$$
\frac{\angle v_{t} 0 v_{t+1}}{2}=\frac{\delta^{t}-\delta^{t+1}}{2}=\frac{1-\delta}{2} \delta^{t} .
$$

Analogously, the angle between $R_{i, t+1}$ and $R_{i, t+2}$ is equal to $\frac{1-\delta}{2} \delta^{t+1}$. Hence, all ratios $\frac{\alpha_{s}}{\alpha_{s+1}}$ are equal to $\delta$, and the conditions of Lemma 5 are satisfied.

This completes the proof of Theorem 2 .

Acknowledgement. The author is grateful to Mark van Kreveld for calling his attention to the problem addressed in this paper, and to Radoslav Fulek, Fabrizio Frati, and Deniz Sarıöz for valuable discussions, and Deniz Sarıöz also for coding the figures in TikZ.

\section{References}

[BGR04] Barequet, G., Goodrich, M.T., Riley, C.: Drawing planar graphs with large vertices and thick edges. J. Graph Algorithms Appl. 8, 3-20 (2004)

[CE92] Chazelle, B., Edelsbrunner, H.: An optimal algorithm for intersecting line segments in the plane. J. ACM 39, 1-54 (1992)

[CS89] Clarkson, K.L., Shor, P.W.: Application of random sampling in computational geometry, II. Discrete \& Computational Geometry 4, 387-421 (1989)

[DEKW06] Duncan, C.A., Efrat, A., Kobourov, S.G., Wenk, C.: Drawing with fat edges. Int. J. Found. Comput. Sci. 17, 1143-1164 (2006)

[ES35] Erdös, P., Szekeres, G.: A combinatorial problem in geometry. Compositio Mathematica 2, 463-470 (1935)

[GRS90] Graham, R., Rothschild, B., Spencer, J.H.: Ramsey Theory. John Wiley and Sons, New York (1990)

[K09] van Kreveld, M.: Bold graph drawings. In: Proc. Canadian Conference on Computational Geometry, CCCG 2009 (2009), http://cccg.ca/proceedings/2009/cccg09_31.pdf Also Computational Geometry: Theory \& Applications (to appear)

[M88] Mulmuley, K.: A fast planar partition algorithm, I. In: Proc. 29th FOCS, pp. 580-589 (1988)

[N04] North, S.C.: Drawing Graphs with Neato (2004), http://www.graphviz.org/Documentation/neatoguide.pdf

[R30] Ramsey, F.P.: On a problem of formal logic. Proc. London Math. Soc. Series 30(2), 264-286 (1930) 